\title{
CapTable and CapShelf - Unobtrusive Activity Recognition Using Networked Capacitive Sensors
}

\author{
Raphael Wimmer ${ }^{1}$, Matthias Kranz ${ }^{1}$, Sebastian Boring ${ }^{2}$, Albrecht Schmidt ${ }^{1}$ \\ Embedded Interaction Research Group ${ }^{1}$, Fluidum Research Group ${ }^{2}$ \\ University of Munich \\ Amalienstrasse 17, 80333 Munich, Germany \\ \{raphael,matthias,albrecht\}@hcilab.org,sebastian.boring@ifi.lmu.de
}

\begin{abstract}
In this paper we introduce two pieces of activitysensing furniture using networked capacitive sensors. CapTable and CapShelf are two example applications for activity detection and context acquisition realized with the CapSensing Toolkit [1]. Both instances are representatives of a greater class of scenarios where networked sensing can compete with other technologies. CapTable is a simple wooden table equipped with capacitive sensors. Hand and body motion can be tracked above and around the table with high resolution. Additionally, conductive and nonconductive objects can be tracked and discriminated. The same features apply to CapShelf, a shelf that can monitor where people are reaching, and partially track the amount of items still in the shelf. We argue, that capacitive sensors provide huge benefits for real-world, privacy-sensitive, and unobtrusive data acquisition and implicit human-computer interaction.
\end{abstract}

\section{INTRODUCTION}
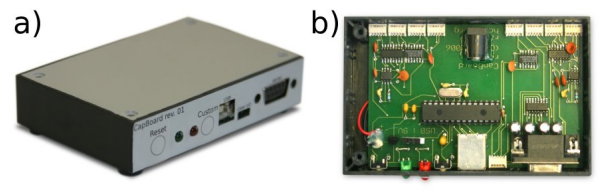

c)

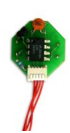

Fig. 1. CapBoard (a) hardware: a microcontroller (b) controls and reads out up to eight small sensors (c)

Capacitive Sensors measure the distance between an electrode and a grounded conductive element. The working principle is simple. The parallel-plate capacitor formed by electrode and object provides a certain electrical capacitance. The capacitance depends - among other variables - on the distance between them. If other variables (like humidity or object size) are controlled, measuring this capacitance provides information about the distance between electrode and object.

The paper is structured as follows. First, we briefly revisit related work with a focus on application scenarios for capacitive sensing. For a comprising, in-depth discussion of the basics of capacitive sensing, we would like to refer the reader to our work describing the CapSensing Toolkit [1]. We then present the application side of the extended CapToolkit as novel part of this work. Finally, we present two new application scenarios for networked capacitive scening systems.

\section{RELATED WORK}

We start with a discussion of existing research in Capacitive Sensing. We look at previous solutions for activity-sensing furniture. Finally we explain, what discerns CapTable and CapShelf from the mentioned work, and why it enables a new range of applications.

\section{A. Applications of Capacitive Sensing}

Most research in Capacitive Sensing has been done at the MIT Media Lab. Zimmerman et al. [2] have coined the term Electric Field Sensing (EFS) for a group of field-sensor technologies, which includes Capacitive Sensing. Paradiso et al. [3] have built several musical instruments utilising EFS. Lee et al. demonstrated an input device for 3D modelling which uses EFS. Dietz et al. [4] built DiamondTouch, a table with a large integrated touchpad. It provides very high resolution finger tracking and can discern multiple users. Rekimoto incorporated EFS hardware into several input devices like keypads or game controllers [5]. SmartSkin [6] enhanced the DiamondTouch concept by also tracking the distance between table top and hand, thus recognising e.g. a hand hovering over the table. Specially tagged objects can also be tracked and discriminated.

\section{B. Sensor-Enhanced Furniture}

Instrumented furniture is greatly suited for tracking movement or events in everyday scenarios. Metzger [7] presented a store shelf that could track the amount of items in it by using load sensors. Schmidt et al. tracked the position of objects on a table with load sensors. Butz et al. [8] used a video camera mounted on a steerable projector for detecting barcode-tagged objects in a room. Kranz et al. [9] were able to discriminate e.g. the cutting sounds of various vegetable on a table.

\section{From Explicit to Implicit Interaction}

Capacitive Sensing or EFS has been widely employed for explicit Human-Computer Interaction, e.g. for gesture interfaces, musical instruments, or for enhancing other input devices. While DiamondTouch and SmartSkin present examples of instrumented furniture, they are intended for explicit interaction. In the context of instrumented environments, sensorenhanced furniture, that detects a user's actions and reacts to it, is of major importance.

In the following sections we explain how real application scenarios using furniture enhanced with capacitive sensors might look like. We highlight the potentials with a specific focus for implicit interaction. Implicit interaction is much more 
interesting for these setting as they do not require any explicit action or knowledge about the existence of the user interface. This is especially suitable for public scenarios where untrained users are the prime users of this invisible technology.

\section{CAPTOOLKIT}

CapToolkit is a newly developed hardware and software platform for Capacitive Sensing. It consists of sensor hardware (CapBoard), simple middleware (CapBoard Bridge) and software tools and demo applications.

\section{A. Sensor Hardware}

CapBoard (Figure 1) supports up to eight sensor channels. Each sensor, measuring $2 \mathrm{~cm} \times 2 \mathrm{~cm} \times 1 \mathrm{~cm}$, generates a signal with a frequency proportional to the capacitance it measures. This frequency is then measured by the CapBoard. CapBoard filters and reprocesses these values and outputs them via 'raw' USB Interrupt transfers. CapBoard also receives commands via USB and controls sensor activation, as well as additional, user-defined I/O channels. For details see [1].

\section{B. Networking Middleware}

CapBoard is connected to a host computer via USB. This has several advantages over e.g. a wireless or ethernet connection:

- integrated $5 \mathrm{~V}$ power supply over the USB bus

- USB subsystem already integrated in microcontroller, no additional components are needed

- device, by its PIC microcontroller, can implement various USB device classes, e.g. act as a mouse to the OS

- communication over widely supported, reliable interface (compared to various wireless communication standards)

CapBoard can also be accessed over TCP/IP. For this, a simple USB-to-TCP/IP bridging tool needs to be run on the host computer. This tool, CapBoard Bridge copies CapBoard's output from USB to TCP sockets and vice versa. CapBoard Bridge supports multiple CapBoard devices connected to one computer. Multiple clients can connect to CapBoard bridge simultaneously and receive sensor data. CapBoard Bridge is currently limited to Linux. It can easily be cross-compiled for embedded Linux systems, e.g. the Sharp Zaurus PDA line. These embedded systems can then deliver sensor data over a variety of network protocols. This enables multiple CapBoards to be combined for larger-scale applications.

\section{Software Tools}

Middleware bindings The Player/Stage/Gazebo (P/S/G) project produces tools for rapid development of sensor and actuator applications. Originally developed for and by the robotics community, $\mathrm{P} / \mathrm{S} / \mathrm{G}$ has become a de facto standard [10] and is in use in the context of pervasive computing e.g. for the AwareKitchen research project presented at INSS 2007.

$\mathrm{P} / \mathrm{S} / \mathrm{G}$ makes use of an enhanced client/server model, featuring auto-discovery mechanisms as well as permitting servers and clients to communicate between them in a heterogeneous network. Due to the standards that $\mathrm{P} / \mathrm{S} / \mathrm{G}$ imposes on its architectural and transport layer, and because of the fact that $\mathrm{P} / \mathrm{S} / \mathrm{G}$ was designed to be language and platform (POSIX) independent, programmers have been able to code their clients in a large variety of programming languages [11].

By providing bindings to this common and extensively used and well developed platform we hope to leverage the usage of our CapSensing toolkit in other scenarios and by other researchers.

CapBoard Analyzer is a Java application visualising the sensor values. It connects to the socket provided by CapBoard Bridge and plots the received data on the screen. This tool also allows setting CapBoard parameters like acquisition time, filtering, or sensor selection. Additional software using CapBoard data can be easily written in a variety of programming languages, as all communication happens with a plain-text protocol with short and simple commands over TCP/IP. Adhering to a standardised communication protocol was a design decision with regards to the re-usage of the CapToolkit within others than the initial settings envisioned by us.

\section{EXAMPLE ScEnARIOS AND APPlication AREAS}

\section{A. CapTable}

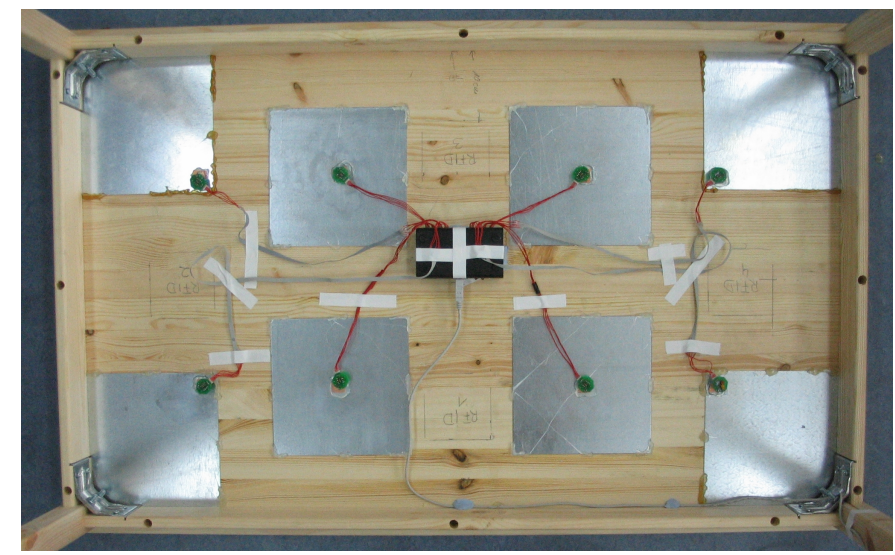

Fig. 2. The CapTable is equipped with eight sensor plates, arranged in a $4 \times 2$ grid. Due to their large size of $20 \mathrm{~cm} \times 20 \mathrm{xcm}$ the sensor plates are very immune to weak external electric fields. For many applications, smaller sensor plates are sufficient.

CapTable (Fig. 2) is a wooden table equipped with eight steel sensor plates are affixed to it from underneath. Each plate measures $20 \times 20 \mathrm{~cm}$ and is attached to a sensor circuit, which in turn is connected to a CapBoard. The CapBoard is connected to a PC which offers the sensor readings over TCP/IP. The whole setup is closely integrated into the AwareKitchen research project. A P/S/G setup (see Section III-C) combines CapTable data with other sensor data from door switches, laser scanners, or RFID readers.

CapTable's sensors allow tracking of hand gestures up to 70 $\mathrm{cm}$ above the table. Additionally, movement in the horizontal proximity of the table can be tracked reliably. This allows e.g. tracking, if and where users are standing around the table. For tracking a single hand above the table, its position can be triangulated from the sensor values. More than one hand can be tracked simultaneously, if each hand's movement is confined to a distinct area of the table top (Figure 3). This is e.g. easily possible in a vis-a-vis scenario when people are 
jointly collaborating, e.g. laying out presentation material. The augmentation is invisible and much cheaper compared to other solutions and does not require a camera.

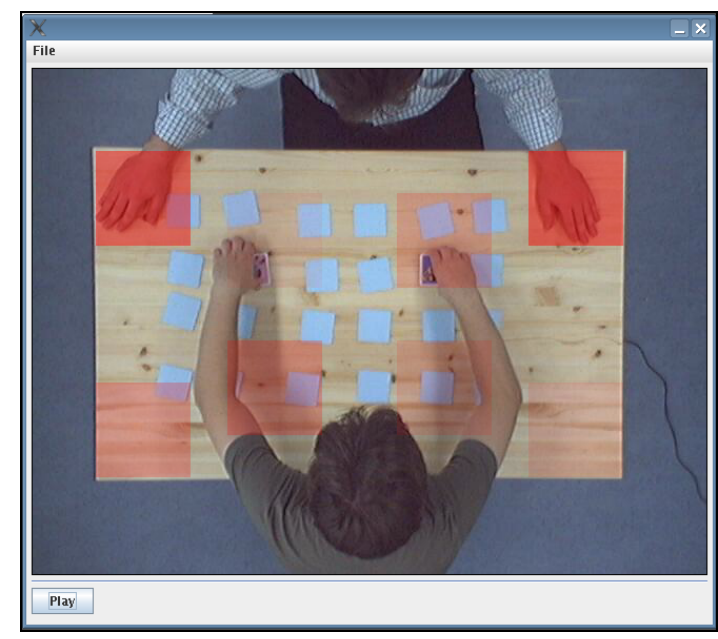

Fig. 3. Two men are playing Memory. A preliminary tracking algorithm already allows detection of hand positions. By interpolating hand positions from sensor readings of the different plates, a much higher resolution will probably be reached. The overlay is from a screenshot of a JMF-based visualisation-only tool with a short lag. This explains e.g. the missing highlighted area around the left hand of the person sitting at the bottom side of the table.

Recognising gestures from multiple users over the whole table - a topic which we have not pursued yet - is harder, because sensors cannot discriminate different user's hands. To enable multi-user gesture tracking, tracking state-information and solving more complex inverse problems will be necessary.

In addition to the CapTable in the AwareKitchen research project, we are currently converting an office desk at our research unit to a CapTable. Our goal is to generate and log long term activity data of a user in a common office scenario. We hope to be able to reliably discriminate different actions like placing a phone call, typing, or filing and retrieving documents. A networked camera above the desk provides reference tracking data used to train a classifier and to verify recognised patterns. After the training, the camera will be removed as the CapBoard technology does not require it and privacy issues shall not be raised in any out-of-the-lab scenario.

In addition to tracking human bodies and hand gestures, CapTable's sensors also detect capacitance changes caused by non-conductive objects, like e.g. glass or porcelain. Their dielectric properties change the electric field emitted by the sensors. For a proof of concept, an empty soda water bottle rolling over the table caused significant changes in sensor data (Figure 4). Additionally, we were able to discriminate three of four nearly identical empty coffee mugs from the sensor values. The mugs were repeatedly placed onto roughly the same spot on the table. The sensor readings were logged. While two cups had the same effect on the sensors, the two other cups caused significantly different sensor readings. These effects are a lot smaller than the capacitance changes caused by the human body. In reality, it may be hard to recognise such effects. However, for specific applications sensor layout and detection algorithms could be customised to eliminate interfering effects.

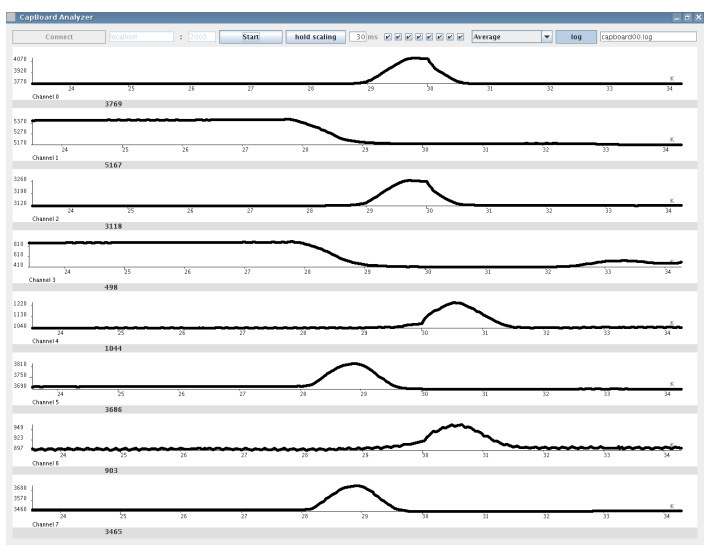

Fig. 4. An empty glass bottle, though not conductive, can be reliably tracked on CapTable. The plot shows sensor data generated by a bottle rolling from left to right over the table, and falling off it.

\section{B. CapShelf}

CapShelf is a standard wooden shelf instrumented with capacitive sensors. It allows tracking of hand movement inside the shelf. This information can be used for inferring objects on the shelf the user touches or takes. Depending on the material the objects are made of, CapShelf can even keep track of the number of items on the shelf. For example, liquid-filled bottles or metallic objects are recognised excellently. Thus it is possible to distinguish objects taken from the shelf. This is especially interesting for high-priced objects like parfumes. One could imagine of starting an audio or video advertisement for a specific parfume when a customer takes out a parfume for closer inspection. The solution here is much cheaper and faster compared e.g. to an array of RFID readers.

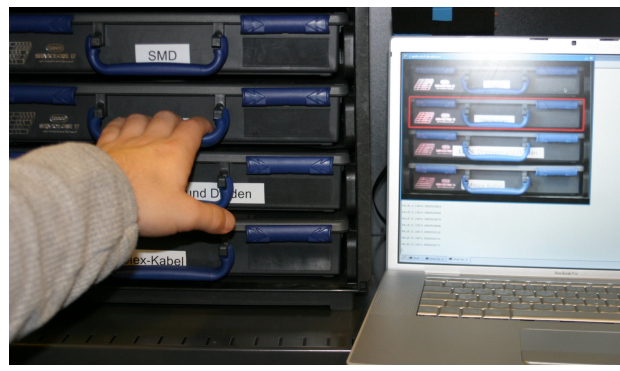

Fig. 5. CapShelf is able to track a hand inside it. For this small sensor electrodes of $10 \times 10 \mathrm{~cm}$ are attached to the boards. In this photo, CapShelf is used to detect which drawer the user is opening.

For tracking a hand inside the shelf, the same algorithm that has been used for the Thracker [12] is applied. In fact, CapShelf (see Fig. 5) is expanding the Thracker idea to facilitate new usage scenarios. CapShelf easily allows tracking a hand with a resolution of $5 \mathrm{~cm}$ and better within a compartment.

\section{Real World Application Scenarios}

While CapTable and CapShelf currently only exist as laboratory prototypes, we strongly believe in their usefulness in real-life applications. Initial tests on both systems have been conducted that showed their application potential. Capacitive 
Sensing offers many properties unique to this sensor technology that predestinates it for many application areas outside research labs:

- unobtrusiveness: Unlike cameras, IR sensors, or laser scanners, capacitive sensors do not need a line-of-sight. Sensors can be embedded in arbitrary housings or hidden behind walls as long as these are non-conductive. This greatly facilitates usage in living rooms and offices.

- robustness: Our sensor systems do not have any moving or sensitive parts, nor has any part of it to be exposed directly to the user. This makes it especially suitable for public spaces where vandalism is a potential problem.

- data-efficiency: The information a capacitive sensor provides is determined by its size, shape, and placement. Thus sensors can be easily adapted for special sensing purposes, so that they provide exactly the data needed. Sensor data is very low-bandwidth, but can be scaled during sensor operation.

- privacy-awareness: Capacitive sensors are especially suited for areas where privacy concerns apply, be it offices [13], public spaces, or homes. While able to provide very high-resolution distance readings, they are largely ignorant to the hand, body, or object they are tracking.

- affordability: The price of our capacitive sensors is very low. Even in small (research) quantities a sensor costs less than two Euro, a CapBoard less than 30 Euro. Thus massive sensor overprovisioning is feasible, where resolution and reliability are increased.

These properties make CapTable and CapShelf perfectly suited for many different scenarios. For example, CapTable allows tracking of assembly procedures, where the different parts of a product are taken from different places on the table or from CapShelf. Thus it would be possible to optimise component placement, detect errors, or create (morally and legally questionable) activity patterns of the assembling person.

By detecting the amount of items on the shelf or table, logistics software can determine the need for delivery of additional supplies. Long-term acquisition of statistical data is feasible, as capacitive sensors are data-efficient and privacyaware and require no maintenance.

Another interesting scenario are indoor scenarios to unobtrusively and privacy-secure detect implicit contexts, e.g. the number of persons and their actions. Wren et al. [13] proposed passive IR sensors for this task. Obtaining only binary values they infer the number of persons and their routes through an office building. This is especially interesting as this requires little effort regarding the installation costs. We think that we could obtain the same information with our capacitive sensing approach. Additionally, as we do not get only binary values, we believe that we could even extend this scenario by interesting aspects. Sensor plates can easily mounted below a carpet or next to walls.

CapTable also allows e.g. sociologists to conduct long-term studies on habits, living, and activities of people. CapTable preserves the people's privacy and blends with the other furniture. It can provide data on table usage (e.g. cooking, eating, reading) or general household activity.

\section{Future WORK AND CONCLUSIONS}

We presented new scenarios for the CapToolkit [1], where capacitive sensors can provide significant and useful data for implicit human-computer interaction and provides a cheaper solution compared to similar approaches. We are currently working on long-term data acquisition at an office desk equipped with capacitive sensors. The data will be used for extracting activity patterns while at the same time accounting for user privacy. The data will be made available to other researchers to run their pattern matching algorithms on them. We plan to equip an existing instrumented room with capacitive sensors, improve position detection algorithms, and apply the technology to new scenarios.

The complete toolkit, containing schematics, source code and documentation, will be put under a free license. Information about capacitive sensing and the toolkit, as well as the toolkit itself, will be published at http://www.capsense.org soon.

\section{REFERENCES}

[1] R. Wimmer, M. Kranz, S. Boring, and A. Schmidt, "A capacitive sensing toolkit for pervasive activity detection and recognition," in Percom 2007.

[2] T. G. Zimmerman, J. R. Smith, J. A. Paradiso, D. Allport, and N. Gershenfeld, "Applying electric field sensing to human-computer interfaces," in CHI '95: Proceedings of the SIGCHI conference on Human factors in computing systems. New York, NY, USA: ACM Press/Addison-Wesley Publishing Co., 1995, pp. 280-287.

[3] J. Paradiso and N. Gershenfeld, "Musical applications of electric field sensing," pp. 69 - 89, 1997.

[4] P. Dietz and D. Leigh, "Diamondtouch: a multi-user touch technology," in UIST '01: Proceedings of the 14th annual ACM symposium on User interface software and technology. New York, NY, USA: ACM Press, 2001, pp. 219-226.

[5] J. Rekimoto, "Gesturewrist and gesturepad: Unobtrusive wearable interaction devices,' in ISWC '01: Proceedings of the 5th IEEE International Symposium on Wearable Computers. IEEE Computer Society, 2001, p. 21.

[6] - "Smartskin: an infrastructure for freehand manipulation on interactive surfaces," in CHI '02: Proceedings of the SIGCHI conference on Human factors in computing systems. New York, NY, USA: ACM Press, 2002, pp. 113-120.

[7] C. Metzger, "Indirect object-sensing technology to prevent outof-stock at retail-level," in In Proceedings of the International Workshop on Smart Object Systems in Conjunction with the Seventh International Conference on Ubiquitous Computing, September 2005. [Online]. Available: http://ubicomp.lancs.ac.uk/workshops/sobs05/ papers/Metzger, $1 \% 20$ Christian.pdf

[8] A. Butz, M. Schneider, and M. Spassova, "Searchlight - a lightweight search function for pervasive environments." in Pervasive, ser. Lecture Notes in Computer Science, A. Ferscha and F. Mattern, Eds., vol. 3001. Springer, 2004, pp. 351-356.

[9] M. Kranz, R. B. Rusu, A. Maldonado, A. Schmidt, and M. Beetz, "Sensing technologies and the player-middleware for context-awareness in kitchen environments," in INSS 2007.

[10] T. H. Collett, B. A. MacDonald, and B. P. Gerkey, "Player 2.0: Toward a Practical Robot Programming Framework," in Proceedings of the Australasian Conference on Robotics and Automation (ACRA 2005), December 2005

[11] Player/Stage/Gazebo: Free Software tools for robot and sensor applications, "http://playerstage.sourceforge.net," 2001.

[12] R. Wimmer, P. Holleis, M. Kranz, and A. Schmidt, “Thracker - using capacitive sensing for gesture recognition," icdcsw, vol. 0, p. 64, 2006

[13] C. R. Wren and E. M. Tapia, "Toward scalable activity recognition for sensor networks." in LoCA, 2006, pp. 168-185. 